\title{
Investigation of HZETRN 2010 as a Tool for Single Event Effect Qualification of Avionics Systems
}

\author{
Kristina Rojdev ${ }^{1}$, William Atwell ${ }^{2}$, Paul Boeder ${ }^{2}$, and Steve Koontz ${ }^{1}$ \\ ${ }^{1}$ NASA Johnson Space Center, Houston, TX 77059 \\ ${ }^{2}$ Boeing Research and Technology, Houston, TX 77059
}

\begin{abstract}
$\underline{\text { Abstract }}$
NASA's future missions are focused on deep space for human exploration that do not provide a simple emergency return to Earth. In addition, the deep space environment contains a constant background Galactic Cosmic Ray (GCR) radiation exposure, as well as periodic Solar Particle Events (SPEs) that can produce intense amounts of radiation in a short amount of time. Given these conditions, it is important that the avionics systems for deep space human missions are not susceptible to Single Event Effects (SEE) that can occur from radiation interactions with electronic components. The typical process to minimizing SEE effects is through using heritage hardware and extensive testing programs that are very costly.

Previous work by Koontz, et al. [1] utilized an analysis-based method for investigating electronic component susceptibility. In their paper, FLUKA, a Monte Carlo transport code, was used to calculate SEE and single event upset (SEU) rates. This code was then validated against in-flight data. In addition, CREME-96, a deterministic code, was also compared with FLUKA and inflight data. However, FLUKA has a long run-time (on the order of days), and CREME-96 has not been updated in several years.

This paper will investigate the use of HZETRN 2010, a deterministic transport code developed at NASA Langley Research Center, as another tool that can be used to analyze SEE and SEU rates. The benefits to using HZETRN over FLUKA and CREME-96 are that it has a very fast run time (on the order of minutes) and has been shown to be of similar accuracy as other deterministic and Monte Carlo codes when considering dose [2, 3, 4]. The 2010 version of HZETRN has updated its treatment of secondary neutrons and thus has improved its accuracy over previous versions. In this paper, the Linear Energy Transfer (LET) spectra are of interest rather than the total ionizing dose. Therefore, the LET spectra output from HZETRN 2010 will be compared with the FLUKA and in-flight data to validate HZETRN 2010 as a computational tool for SEE qualification by analysis. Furthermore, extrapolation of these data to interplanetary environments at $1 \mathrm{AU}$ will be investigated to determine whether HZETRN 2010 can be used successfully and confidently for deep space mission analyses.
\end{abstract}




\section{$\underline{\text { References }}$}

[1] S. Koontz, B. Reddell and P. Boeder, "Calculating Spacecraft Single Event Environment with FLUKA," in Radiation Effects Data Workshop, Las Vegas, 2011.

[2] Z. Lin, J. J. Adams, A. Barghouty, S. Randeniya, R. Tripathi, J. Watts and P. Yepes, "Comparisons of Several Transport Models in their Predictions in Typical Space Radiation Environments," Advances in Space Research, vol. 49, pp. 797-806, 2012.

[3] J. H. Heinbockel, T. C. Slaba, S. R. Blattnig, R. K. Tripathi, L. W. Townsend, T. Handler, T. A. Gabriel, L. S. Pinsky, B. Reddell, M. S. Clowdsley, R. C. Singleterry, J. W. Norbury and F. F. Badavi, "Comparison of the transport codes HZETRN, HETC and FLUKA for a solar particle event," Advances in Space Research, vol. 47, no. 6, pp. 1079-1088, 2011.

[4] J. H. Heinbockel, T. C. Slaba, R. K. Tripathi, S. R. Blattnig, J. W. Norbury, F. F. Badavi, L. W. Townsend, T. Handler, T. A. Gabriel, L. S. Pinksy, B. Reddell and A. R. Aumann, "Comparison of the transport codes HZETRN, HETC and FLUKA for galactic cosmic rays," Advances in Space Research, vol. 47, no. 6, pp. 1089-1105, 2011.

To be submitted AIAA SPACE 2014 Conference Manchester Grand Hyatt Hotel

San Diego, CA 92101

4-7 August 2014 